Artigo original

Raildo da Silva Coqueiro ${ }^{1,4}$

Adriana Alves Nery ${ }^{1}$

Zoraide Vieira Cruz ${ }^{1}$

Cloud Kennedy Couto de Sá ${ }^{2,3}$

\title{
FATORES ASSOCIADOS AO SOBREPESO EM ADULTOS ACOMPANHADOS POR UMA UNIDADE DE SAÚDE DA FAMÍLIA
}

\author{
FACTORS ASSOCIATED TO THE OVERWEIGHT IN ADULTS ASSISTED BY A \\ FAMILY HEALTH UNIT
}

\begin{abstract}
RESUMO
O objetivo do estudo foi verificar a freqüência e fatores associados ao sobrepeso em adultos acompanhados por uma Unidade de Saúde da Família (USF), na cidade de Jequié, Bahia, Brasil. O desenho foi transversal em amostra de 101 adultos $\geq 18$ anos de idade, cadastrados e distribuídos proporcionalmente entre as sete microáreas da USF, sendo $75(74,0 \%)$ do sexo feminino e $26(26,0 \%)$ do masculino. Com sobrepeso foram considerados aqueles que apresentaram índice de massa corporal (IMC) $\geq 25 \mathrm{~kg} / \mathrm{m}^{2}$. Calculou-se a freqüência do sobrepeso no total e por variáveis associadas (idade, sexo, ocupação, escolaridade, número de refeições diárias, tabagismo, etilismo, hipertensão arterial, diabetes, dislipidemias e nível de atividade física habitual - NAFH). As associações foram verificadas utilizando o teste do qui-quadrado, ao nível de significância de $5 \%(p \leq 0,05)$. A freqüência do sobrepeso foi de $28,0 \%$, sendo mais prevalente apenas em indivíduos que não consomem bebidas alcoólicas, embora a comparação entre hipertensos e não hipertensos tenha se apresentado limítrofe $(p=0,052)$. Os resultados deste estudo sugerem outras investigações sobre o efeito do consumo de bebidas alcoólicas sobre o estado nutricional de adultos e implementação de estratégias de vigilância nutricional na USF, com atenção especial nos hipertensos.
\end{abstract}

Palavras-chave: Estilo de vida; Fatores de risco; Índice de massa corporal; Estado nutricional.

\begin{abstract}
The objective of this study was to investigate the factors associated with overweight in adults and the frequencies of these factors. The sample comprised 101 adults, all aged $\geq 18$ years, $75(74.0 \%)$ females and $26(26.0 \%)$ males, registered at a Family Health Center (FHC) in the town of Jequié, and distributed proportionally throughout the FHC's seven sub-areas. Patients were defined as overweight if their body mass index (BMI) was $\geq 25 \mathrm{~kg} / \mathrm{m}^{2}$. The proportion of overweight people was calculated for the whole sample and for each of the associated variables (age, sex, occupation, educational level, number of meals per day, smoking, alcoholism, arterial hypertension, diabetes, dyslipidemia and habitual level of physical activity). The chi-square test was applied to test associations between the variables and the proportion of overweight to a significance level of $5 \%(p \leq 0.05)$. the overall frequency of overweight was $28.0 \%$, and this was more prevalent only among individuals who do not consume alcoholic beverages, although the comparison between those with and without hypertension was borderline $(p=0.052)$. The results of this study suggest a need for further investigation into the effect of alcoholic beverages on the nutritional status of adults and for implementation of nutritional vigilance strategies at this $\mathrm{FHC}$, with special attention given to hypertense patients.
\end{abstract}

Key words: Lifestyle. Risk factors. Body mass index. Nutritional status.

1 Universidade Estadual do Sudoeste da Bahia. Departamento de Saúde, Jequié, BA, Brasil.

2 Universidade Estadual de Feira de Santana, Feira de Santana, BA, Brasil

3 Fundação Bahiana para Desenvolvimento das Ciências. Programa de Pós-Graduação em Medicina e Saúde Humana Salvador, BA, Brasil.

4 Bolsista CNPq (GM 132614/2007-3). 


\section{INTRODUÇÃO}

A sociedade moderna tem vivenciado, nas últimas décadas, um complexo processo de mudanças nos padrões alimentares e nutricionais, nos perfis demográfico e sócio-econômico' ${ }^{1}$. A transição nutricional, que se caracteriza pela inversão da razão entre desnutrição e obesidade, na qual grande parte da população mundial vive, é um bom exemplo. No Brasil, algumas décadas atrás, a desnutrição excedia a obesidade em uma vez e meia, enquanto a partir da década de 1990 a obesidade passou a exceder a desnutrição em mais de duas vezes ${ }^{2}$.

Esse fato tem mostrado que o problema da escassez tem sido rapidamente substituído pelo do excesso alimentar, resultando em desequilíbrio entre a ingestão e o gasto energético, o que segundo Castro et al. ${ }^{3}$, é o principal determinante de um dos maiores problemas atuais de saúde pública: o sobrepeso. Tal processo vem acontecendo de maneira diferenciada em países, regiões e grupos sociais diversos ${ }^{4-7}$.

Desse modo, diversos estudos epidemiológicos foram realizados, procurando relacionar sobrepeso com comportamentos relacionados à saúde, a exemplo da prática de atividades físicas, hábitos alimentares, controle de stress, dentre outros ${ }^{3,6-15}$. Tais estudos revelam que a ocorrência de sobrepeso está associada com a qualidade da alimentação, com as atividades diárias e o ambiente físico e social.

Há atualmente uma diversidade de métodos listados na literatura para avaliar o sobrepeso num contexto populacional ou na prática clínica ${ }^{2}$. O índice de Massa Corporal (IMC), calculado por meio de medidas antropométricas (peso/estatura ${ }^{2}$ ), tem sido utilizado com freqüência em levantamentos populacionais, para determinação do sobrepeso. Tal fato se deve à praticidade, custo aceitável, por refletir com certa precisão o estado nutricional dos indivíduos e por ter uma grande quantidade de bancos de dados disponíveis para efeito de comparação $0^{4,8,16}$. Além disso, estudos recentes demonstraram que inadequação nutricional (baixo peso e sobrepeso) é associado a risco aumentado de morte 17,18 .

Apesar do Programa de Saúde da Família (PSF) ser visto pelo Ministério da Saúde (MS) como a mais importante mudança estrutural já ocorrida na saúde pública do Brasil ${ }^{19}$, e do grande número de unidades de Saúde da Família (USFs) espalhadas em todo território nacional, poucos estudos tiveram a preocupação de identificar a tendência para a inadequação nutricional em nível local. Investigações desse tipo podem contribuir para o gerenciamento de estratégias melhor direcionadas às necessidades específicas de cada localidade.

Esse trabalho teve como objetivo verificar a freqüência e fatores associados ao sobrepeso em adultos acompanhados por uma USF na cidade de Jequié, Bahia, Brasil.

\section{PROCEDIMENTOS METODOLÓGICOS}

Estudo transversal realizado em Jequié, em 2004, com usuários do PSF cadastrados na USF Dr. Milton
Rabello I. Adotou-se como critérios para a seleção da USF: (1) não estar instalada na zona rural; (2) equipe que atendesse a recomendação do Ministério da Saúde (MS) de acompanhamento de 600 a 1000 famílias $^{19}$; (3) maior tempo de implantação no PSF. Dessa forma, a equipe que atendeu todos os critérios foi a acima referida, que fica situada em um bairro carente da cidade.

\section{Amostra}

A amostra foi intencional, sendo feita seguindo as seguintes etapas: (1) Sorteio aleatório simples de $101(10 \%)$ famílias cadastradas no prontuário familiar da USF, proporcional as sete microáreas da unidade, sendo realizado pelo pesquisador na presença de cada agente comunitário de saúde (ACS) responsável pelas respectivas microáreas; (2) determinação de que apenas um único membro (o primeiro a receber o pesquisador e o ACS) de cada família, com idade igual ou superior a 18 anos, participaria do estudo. Quando não se encontrava na residência selecionada nenhum indivíduo com idade adequada ou este se recusava a participar da pesquisa, uma nova família era sorteada. Dessa forma, a amostra foi constituída por 101 adultos com idade entre 18 a 88 anos, sendo 75 do sexo feminino e 26 do sexo masculino.

\section{Variáveis de estudo}

As variáveis do estudo foram: sobrepeso (variável dependente), idade, sexo, ocupação, escolaridade, número de refeições diárias, tabagismo, consumo de álcool, hipertensão arterial, diabetes, dislipidemias e nível habitual de atividade física (variáveis independentes).

\section{Coleta dos dados}

Um entrevistador de campo foi devidamente treinado para todas as etapas do trabalho. Para testes e correções dos instrumentos e técnicas de medidas antropométricas, inclusive da dinâmica do trabalho de campo, 10 famílias (10 participantes) foram visitadas e as entrevistas e medidas, realizadas seguindo toda metodologia proposta. Todos os participantes da pesquisa foram entrevistados em domicílio para coleta dos dados. Com relação ao diagnóstico de sobrepeso, foi utilizado o IMC, adotando-se os seguintes procedimentos para aferição das medidas: (1) Massa corporal - Esta medida foi realizada em balança portátil marca Plenna (EUA) de $150 \mathrm{~kg}$ de capacidade e sensibilidade de $1 / 2 \mathrm{~kg}$ com o indivíduo vestido com o mínimo possível de roupa e descalço; (2) Estatura - Para realizar a medida, fixava-se uma fita métrica, com sensibilidade de $0,1 \mathrm{~cm}$, em local adequado, como parede ou batente de porta, sem rodapés, formando ângulo reto com o piso. O examinado, descalço, era posicionado nesse local, permanecendo ereto, com pés unidos e com calcanhares, nádegas e cabeça em contato com a parede (ou batente da porta) orientada no plano de Frankfurt (linha imaginária traçada entre o rebordo orbital inferior e o meato auditivo externo e que fica paralela ao solo). Para realizar esta medida 
correspondente, foi colocado um esquadro sobre o topo da cabeça do examinado, de modo que formasse um ângulo de $90^{\circ} \mathrm{com}$ a parede (ou batente da porta), e, logo após, marcou-se esse ponto ao final de uma inspiração. Foram considerados com sobrepeso aqueles que apresentaram IMC $\geq 25 \mathrm{~kg} / \mathrm{m}^{2}$, segundo critérios sugeridos pela Organização Mundial da Saúde ${ }^{20}$.

Para a verificação do nível de atividade física habitual (NAFH) foi utilizado o International Physical Activity Questionnaire - IPAQ (versão curta) ${ }^{21}$. As demais variáveis foram coletadas através de um formulário constituído dos seguintes itens: (1) demografia; (2) hábitos nutricionais; (3) hábitos e costumes sociais; (4) presença de doenças e/ou agravos à saúde.

\section{Procedimentos de análise}

Inicialmente, caracterizou-se cada uma das variáveis nominais como sim ou não. Em seguida, calculou-se a prevalência para as variáveis observadas. Para o IMC especificamente foram considerados dois níveis: (1) eutrófico, formado pelos sujeitos com IMC <25kg/m²; (2) sobrepeso, composto por todos os que apresentaram IMC $\geq 25 \mathrm{~kg} / \mathrm{m}^{2}$. Para caracterização do NAFH, também foram considerados dois níveis:
(1) desejável, formado pelos sujeitos classificados como ativos ou muito ativos; (2) indesejável, composto por todos os que apresentaram classificação insuficientemente ativo ou sedentário.

As variáveis ordinais e nominais foram trabalhadas de forma dicotômica e divididas em estratos, respectivamente: sexo (masculino e feminino), idade (18 a 39 anos, 40 a 59 anos e $>59$ anos), escolaridade (analfabeto/fundamental incompleto, fundamental completo/médio incompleto e médio completo/superior incompleto), ocupação (ativo e inativo), número de refeições diárias ( $<3 \mathrm{e} \geq 3$ ), tabagismo (fumante, ex-fumante e não fumante), consumo de álcool (uso abusivo, uso moderado e não usuário), hipertensão arterial (sim e não), diabetes (sim e não) e dislipidemias (sim ou não).

Para a descrição das características gerais dos sujeitos, foram utilizadas as médias, desvios padrão, valores máximo e mínimo e valores percentuais, enquanto que se descreveu a prevalência do sobrepeso nos diversos estratos das variáveis estudadas pelas freqüências absoluta e relativa.

Nas inferências estatísticas, para identificar a diferença na proporção das variáveis segundo o IMC, foi realizado o teste do qui-quadrado, ao nível

Tabela 1. Descrição da amostra [média \pm desvio padrão (mínimo e máximo) e valores percentuais] conforme variáveis demográficas, antropométricas, comportamentais e de saúde. Jequié-BA, Brasil, 2004.

\begin{tabular}{|c|c|c|c|}
\hline Variáveis & Homens $(n=26)$ & Mulheres $(n=75)$ & $p$ \\
\hline Idade (anos)* & $45,7 \pm 18,1(18-85)$ & $37,9 \pm 18,2(18-88)$ & 0,061 \\
\hline Massa corporal $(\mathrm{kg})^{*}$ & $60,0 \pm 9,6(41,7-79,8)$ & $57,5 \pm 12,0(31,8-84,8)$ & 0,347 \\
\hline Estatura $(m)^{*}$ & $1,66 \pm 0,07(1,51-1,82)$ & $1,55 \pm 0,07(1,42-1,73)$ & $<0,001$ \\
\hline $\operatorname{IMC}\left(\mathrm{kg} / \mathrm{m}^{2}\right)^{*}$ & $21,6 \pm 3,1(16,7-28,9)$ & $23,9 \pm 4,8(15,1-36,2)$ & 0,009 \\
\hline \multicolumn{4}{|l|}{ Ocupação ${ }^{\dagger}$} \\
\hline Ativo & 38,5 & 78,7 & \\
\hline Inativo & 61,5 & 21,3 & $<0,001$ \\
\hline \multicolumn{4}{|l|}{ Escolaridade $^{\dagger}$} \\
\hline Analfabeto./ fund. incompleto & 92,3 & 80,0 & \\
\hline Fund. completo./ médio incompleto & 7,7 & 9,3 & \\
\hline Médio completo / sup. incompleto & - & 10,7 & 0,204 \\
\hline \multicolumn{4}{|l|}{$\mathrm{N}^{\circ}$ de refeições diárias ${ }^{\dagger}$} \\
\hline$<3$ & 32,0 & 12,0 & \\
\hline$\geq 3$ & 68,0 & 88,0 & 0,021 \\
\hline \multicolumn{4}{|l|}{ Tabagismo $^{\dagger}$} \\
\hline Não fumante & 53,8 & 81,3 & \\
\hline Ex-fumante & 46,2 & 2,7 & \\
\hline Fumante & - & 16,0 & $<0,001$ \\
\hline \multicolumn{4}{|l|}{ Consumo de álcool (ethanol, g/dia) ${ }^{\dagger}$} \\
\hline 0 (não consome) & 69,2 & 78,7 & \\
\hline$\leq 30$ (uso moderado) & 15,4 & 18,7 & \\
\hline$>30$ (uso abusivo) & 15,4 & 2,6 & 0,061 \\
\hline \multicolumn{4}{|l|}{$\mathrm{NAFH}^{+}$} \\
\hline Desejável & 65,4 & 60,0 & \\
\hline Indesejável & 34,6 & 40,0 & 0,627 \\
\hline \multicolumn{4}{|l|}{ Hipertensão arterial $^{\dagger}$} \\
\hline Sim & 30,8 & 28,0 & \\
\hline Não & 69,2 & 72,0 & 0,788 \\
\hline \multicolumn{4}{|l|}{ Diabetes $^{\dagger}$} \\
\hline Sim & 7,7 & 2,7 & \\
\hline Não & 92,3 & 97,3 & 0,258 \\
\hline \multicolumn{4}{|l|}{ Dislipidemias $^{\dagger}$} \\
\hline Sim & - & 5,3 & \\
\hline Não & 100,0 & 94,7 & 0,230 \\
\hline
\end{tabular}

Valores contínuos $\left(^{*}\right)$ foram comparados por meio do teste "t" de Student para amostras independentes, e valores percentuais $(\dagger)$, por meio do teste qui-quadrado. 
Tabela 2. Frequência do sobrepeso conforme variáveis demográficas, comportamentais e de saúde. Jequié-BA, Brasil, 2004.

\begin{tabular}{|c|c|c|c|c|c|}
\hline \multirow[t]{2}{*}{ Variáveis } & \multicolumn{2}{|c|}{ Eutrófico $(\%=72,3 ; \mathrm{n}=73)$} & \multicolumn{2}{|c|}{ Sobrepeso $(\%=27,7 ; n=28)$} & \multirow[b]{2}{*}{$\chi^{2}$} \\
\hline & $\%$ & $\mathrm{n}$ & $\%$ & $\mathrm{n}$ & \\
\hline \multicolumn{6}{|l|}{ Idade (anos) } \\
\hline 18 a 39 & 58,0 & 42 & 57,0 & 16 & \\
\hline 40 a 59 & 30,0 & 22 & 18,0 & 5 & \\
\hline$>59$ & 12,0 & 9 & 25,0 & 7 & 0,203 \\
\hline \multicolumn{6}{|l|}{ Sexo } \\
\hline Masculino & 29,0 & 21 & 18,0 & 5 & \\
\hline Feminino & 71,0 & 52 & 82,0 & 23 & 0,262 \\
\hline \multicolumn{6}{|l|}{ Ocupação } \\
\hline Ativo & 68,0 & 50 & 68,0 & 19 & \\
\hline Inativo & 32,0 & 23 & 32,0 & 9 & 0,951 \\
\hline \multicolumn{6}{|l|}{ Escolaridade } \\
\hline Analfabeto. / fund. incom. & 82,0 & 60 & 86,0 & 24 & \\
\hline Fund. comp. / médio incom. & 10,0 & 7 & 7,0 & 2 & \\
\hline Médio comp. / sup. incomp. & 8,0 & 6 & 7,0 & 2 & 0,907 \\
\hline \multicolumn{6}{|l|}{$\mathrm{N}^{\circ}$ de refeições diárias } \\
\hline$<3$ & 15,0 & 11 & 21,0 & 6 & \\
\hline$\geq 3$ & 84,0 & 61 & 79,0 & 22 & 0,462 \\
\hline \multicolumn{6}{|l|}{ Tabagismo } \\
\hline Não fumante & 68,0 & 50 & 89,0 & 25 & \\
\hline Ex-fumante & 3,0 & 2 & - & - & \\
\hline Fumante & 29,0 & 21 & 11,0 & 3 & 0,094 \\
\hline \multicolumn{6}{|l|}{ Consumo de álcool (g/dia) } \\
\hline 0 (não consome) & 70,0 & 51 & 92,0 & 26 & \\
\hline$\leq 30$ (uso moderado) & 23,0 & 17 & 4,0 & 1 & \\
\hline >30 (uso abusivo) & 7,0 & 5 & 4,0 & 1 & 0,045 \\
\hline \multicolumn{6}{|l|}{ NAFH } \\
\hline Desejável & 59,0 & 43 & 68,0 & 19 & \\
\hline Indesejável & 41,0 & 30 & 32,0 & 9 & 0,408 \\
\hline \multicolumn{6}{|l|}{ Hipertensão arterial } \\
\hline $\operatorname{Sim}$ & 23,0 & 17 & 43,0 & 12 & \\
\hline Não & 77,0 & 56 & 57,0 & 16 & 0,052 \\
\hline \multicolumn{6}{|l|}{ Diabetes } \\
\hline Sim & 5,0 & 4 & - & - & \\
\hline Não & 95,0 & 69 & 100,0 & 28 & 0,206 \\
\hline \multicolumn{6}{|l|}{ Dislipidemias } \\
\hline Sim & 3,0 & 2 & 7,0 & 2 & \\
\hline Não & 97,0 & 71 & 93,0 & 26 & 0,307 \\
\hline
\end{tabular}

de significância de $5 \%$ ( $p \leq 0,05)$. Valores contínuos foram comparados através do teste "t" de Student para amostras independentes. Os dados foram analisados através do programa estatístico SPSS, versão 10.0.

O projeto foi apresentado à Comissão de Ética em Pesquisa do Departamento de Saúde da Universidade Estadual do Sudoeste da Bahia (UESB), sendo aprovado na íntegra. Todos os participantes do estudo assinaram termo de consentimento concordando em participar da pesquisa.

\section{RESULTADOS}

Foram entrevistados 101 adultos em sete microáreas da USF selecionada para o estudo, destes, 73 (72,3\%) apresentaram IMC considerado normal. Entretanto, 28 apresentaram IMC com valores superiores ao estabelecido como normal para indivíduos eutróficos, o que representa uma freqüência de sobrepeso de $27,7 \%$.

As características da amostra são apresentadas na Tabela 1. No que se refere às comparações entre sexos, observa-se que existem diferenças entre o sexo masculino e feminino nas médias da estatura $(p<0,001)$ e do IMC ( $p=0,009)$, com os homens apresentando média superior para a primeira variável, enquanto as mulheres para a segunda. Além disso, encontrou-se diferenças nas proporções dos diversos estratos das variáveis ocupação ( $p<0,001)$, número de refeições diárias $(p=0,021)$ e tabagismo $(p<0,001)$, apontando que algum tipo de ocupação, a realização de três ou mais refeições diárias e hábito de fumar foram mais freqüentes em mulheres que em homens. Com relação às demais variáveis, não existem diferenças entre sexos nos diversos estratos (Tabela 1).

As prevalências do sobrepeso em cada um dos estratos das diversas variáveis analisadas, bem como na população total do estudo, encontram-se na Tabela 2. Para consumo de álcool, o sobrepeso predominou entre o grupo dos que não consomem bebidas alcoólicas $(p=0,045)$. Quanto à hipertensão arterial, maior percentual de hipertensos foi observado entre o grupo com sobrepeso, quando comparado ao grupo eutrófico, com a diferença tendo se apresentado limítrofe 
$(p=0,052)$. Nenhuma das demais variáveis do estudo se associou significativamente ao sobrepeso.

\section{DISCUSSÃO}

$\mathrm{Na}$ atual pesquisa, observou-se freqüência de sobrepeso inferior a de muitos trabalhos realizados com populações adultas em diversas regiões do país, como, por exemplo, uma investigação com o objetivo de analisar a prevalência de baixo peso e obesidade em grupamentos sociais da área metropolitana de São Paulo, onde Martins et al. ${ }^{5}$ encontraram percentuais que variaram, de acordo com a idade, entre $26,6 \%$ e $38 \%$. Em amostra de base populacional no município do Rio de Janeiro, a proporção de excesso de peso foi de $43,4 \%$ entre os homens e $39,4 \%$ entre as mulheres ${ }^{16}$.

No geral, os estudos, realizados no Brasil, têm observado prevalências de sobrepeso em adultos de ambos os sexos, que variam de $32,8 \%$ a $53,1 \%$, a depender da região geográfica ${ }^{5,7,9,11,12,14}$.

Apesar de não terem sido encontradas diferenças significativas entre IMC e grupo etário, a leve tendência de maior proporção de sobrepeso em indivíduos mais velhos, parece corroborar a idéia de que a idade pode contribuir para o aumento da massa corporal. Num levantamento realizado por Veggi et al. ${ }^{11}$, com 3.526 funcionários de uma universidade do Rio de Janeiro, com idade entre 22 a 59 anos, cujo objetivo foi testar a hipótese de que a autopercepção inadequada do peso corporal, com ou sem obesidade, estaria associada aos transtornos mentais comuns, verificou-se que, tanto em homens como em mulheres, o IMC se apresentou mais elevado nos indivíduos de maior faixa etária.

Em estudo realizado com 1.041 indivíduos maiores de 20 anos, observou-se que, no grupo etário acima de 40 anos, as mulheres apresentaram IMC significativamente maior $(p<0,001)$ do que os homens ${ }^{5}$. No presente estudo, apesar da freqüência de sobrepeso ter sido levemente mais alta nos indivíduos do sexo feminino, talvez pelo fato da amostra ter sido composta, predominantemente, por pessoas de 18 a 39 anos, tal associação não foi verificada.

Em concordância com os achados de Ell et al. ${ }^{1}$, que realizaram uma investigação com 647 bancários no Rio de Janeiro, com idade entre 22 a 59 anos, foi constatado que o nível de escolaridade parece não ter influência significativa sobre o IMC, uma vez que, tanto no estudo citado, como no nosso, o sobrepeso não se associou a essa variável.

Também não foi encontrada associação entre IMC e o número de refeições diárias, o que reforça a idéia, demonstrada em outros estudos, de que, talvez, o problema de excesso de peso está relacionado muito mais com os tipos de alimentos que as pessoas comem, do que com a quantidade ${ }^{10}$.

A associação significativa entre IMC e etilismo indica que, na população investigada, o fato de as pessoas fazerem uso moderado de bebidas alcoólicas está associado à capacidade de os indivíduos manterem a massa corporal dentro da faixa considerada saudável.
Esse dado foi, de certa forma, surpreendente, uma vez que relato de evento similar não foi encontrado na literatura; ao contrário, estudo recente verificou que, em homens e mulheres, a ingestão diária de álcool decresce com o aumento no IMC ${ }^{18}$.

Em concordância com o que foi observado em outros estudos, o sobrepeso não foi significativamente associado ao nível indesejável de atividade física. De todos os trabalhos analisados, somente um ${ }^{14}$, encontrou associação significativa entre IMC e inatividade física, porém, observada apenas para obesidade (IMC $\geq 30 \mathrm{~kg} /$ $\mathrm{m}^{2}$ ). Quando as análises levaram em consideração o sobrepeso (IMC $\geq 25 \mathrm{~kg} / \mathrm{m}^{2}$ ), nenhuma associação significativa foi observada, tanto em relação ao NAFH global ${ }^{22}$, como para a prática de atividade física de lazer e exercício físico ${ }^{9,11}$.

No presente estudo, a diferença estatística na proporção de hipertensos segundo o IMC se apresentou limítrofe, com maior percentual entre o grupo com sobrepeso. Essa tendência de maior prevalência concorda com os dados encontrados em outros trabalhos ${ }^{23,24}$, que ao contrário deste, verificaram que o aumento no IMC foi significativamente associado à proporção de hipertensão arterial, sugerindo que o excesso de peso pode atuar como fator que influencia no desenvolvimento da hipertensão arterial.

As limitações do presente estudo estão, particularmente, no fato de se empregar um modelo transversal, que, apesar de indicar associações com precisão estatística, não permite ao pesquisador apontar "causa-efeito" com uma aceitável confiabilidade e de se utilizar um formulário em que todas as informações levantadas, com exceção das variáveis antropométricas, foram auto-relatadas. Da mesma forma, pode-se considerar que o tamanho da amostra foi um fator limitante, devido às impossibilidades de se conduzir um trabalho de base populacional, o que pode ter contribuído para o pequeno número de associações significativas.

Os resultados apresentados podem ser utilizados para o diagnóstico que antecede a elaboração de estratégias que visam à participação em programas direcionados à promoção e recuperação da saúde. Levantamentos deste tipo são necessários para monitorar mudanças nos comportamentos associados ao sobrepeso, possibilitando identificar nos subgrupos populacionais os fatores que expõem a maior risco, auxiliando o desenvolvimento de intervenções mais eficientes, que tenham como base a vigilância e promoção da saúde. Recomenda-se outras investigações sobre o efeito do consumo de bebidas alcoólicas sobre o estado nutricional de adultos e implementação de estratégias de vigilância nutricional na USF, com atenção especial nos hipertensos.

\section{CONCLUSÃO}

De acordo com os resultados apresentados no presente estudo, é possível concluir que: o sobrepeso mostrou-se freqüente na amostra estudada (28\%), embora tenha sido inferior a prevalência encontrada 
na maioria dos estudos realizados na população brasileira; o sobrepeso foi mais freqüente apenas em indivíduos que não consomem bebidas alcoólicas, no entanto, verificou-se uma tendência de associação com hipertensão arterial.

\section{REFERÊNCIAS BIBLIOGRÁFICAS}

1. Ell E, Camacho LA, Chor D. Perfil antropométrico de funcionários de banco estatal no Estado do Rio de Janeiro/Brasil: I- Índice de massa corporal e fatores sóciodemográficos. Cad Saúde Pública 1999;15:113-121.

2. Acuña $\mathrm{K}, \mathrm{Cruz} \mathrm{T}$. Avaliação do estado nutricional de adultos e idosos e situação nutricional da população brasileira. Arq Bras Endocrinol Metab 2004;48:345-361.

3. Castro MBT, Anjos LA, Lourenço PM. Padrão dietético e estado nutricional de operários de uma empresa metalúrgica do Rio de Janeiro, Brasil. Cad Saúde Pública 2004;20:926-934.

4. Burlandy L, Anjos LA. Acesso a vale-refeição e estado nutricional de adultos beneficiários do Programa de Alimentação do Trabalhador no Nordeste e Sudeste do Brasil, 1997. Cad Saúde Pública 2001;17:1457-1464.

5. Martins IS, Velásquez-Meléndez G, Cervato AM. Estado nutricional de grupamentos sociais da área metropolitana de São Paulo, Brasil. Cad Saúde Pública 1999;15:71-78.

6. Ciorlia LAS, Godoy MF. Fatores de risco cardiovascular e mortalidade. Seguimento em longo prazo (até 20 anos) em programa preventivo realizado pela medicina ocupacional. Arq Bras Cardiol 2005;85:20-25.

7. Gigante DP, Dias-da-Costa JS, Olinto MTA, Menezes AMB, Macedo S. Obesidade da população adulta de Pelotas, Rio Grande do Sul, Brasil e associação com nível sócio-econômico. Cad Saúde Pública 2006;22:1873-1879.

8. Savio KE, Costa THM, Miazaki E, Schmitz BAS. Avaliação do almoço servido a participantes do programa de alimentação do trabalhador. Rev Saúde Pública 2005;39:148-155.

9. Florindo AA, Latorre MRDO, Tanaka T, Jaime PC, Zerbini CAF. Fatores associados à prática de exercícios físicos em homens voluntários adultos de idosos residentes na Grande São Paulo, Brasil. Rev Bras Epidemiologia 2001;4:105-113.

10. Sichieri L, Castro JFG, Moura AS. Fatores associados ao padrão de consumo alimentar da população brasileira urbana. Cad Saúde Pública 2003;19:547-553.

11. Veggi AB, Lopes CS, Faerstein E, Sichieri R. Índice de massa corporal, percepção do peso corporal e transtornos mentais comuns entre funcionários de uma universidade no Rio de Janeiro. Rev Bras Psiquiatr 2004;26:242-247.

12. Nunes MA, Olinto MTA, Barros FC, Camey S. Influência da percepção do peso e do índice de massa corporal nos comportamentos alimentares anormais. Rev Bras Psiquiatr 2001;23:21-27.

13. Cantos GA, Duarte MFS, Dutra RL, Silva CSM, Waltrick CDA, Balen MG et al. Prevalência de fatores de risco de doença arterial coronária em funcionários de um hospital universitário e sua correlação com estresse psicológico. J Bras Patol Med Lab 2004;40:240-247.

14. Souza LJ, Gicovate-Neto C, Chalita FEB, Reis AFF, Bastos DA, Souto-Filho JTD et al. Prevalência de obesidade e fatores de risco cardiovascular em Campos, Rio de Janeiro. Arq Bras Endocrinol Metab 2003;47:669-676.

15. Thommasen HV, Self B, Grigg A, Zhang W, Birmingham $C L$. The relationship between self-rated health, stress, health care, overall quality of life and weight in a rural population. Eat Weight Disord 2005;10:66-69.

16. Afonso FM, Sichieri R. Associação do índice de massa corporal e a relação cintura/quadril com hospitalizações em adultos do Município do Rio de Janeiro, RJ. Rev Bras Epidemiologia 2002;5:153-163.

17. Jee SH, Sull JW, Park J, Lee S, Ohrr H, Guallar E et al. Body-Mass Index and Mortality in Korean Men and Women. N Engl J Med 2006;355:779-87.

18. Adams KF, Schatzkin A, Harris TB, Kipnis V, Mouw T, Ballard-Barbash R et al. Overweight, Obesity, and Mortality in a Large Prospective Cohort of Persons 50 to 71 Years Old. N Engl J Med 2006;355:763-78.

19. BRASIL. Saúde da Família: uma estratégia para a reorientação do modelo assistencial. Brasília: Ministério da Saúde; 1997.

20. World Health Organization, Obesity: preventing and managing the global epidemic. Geneva: World Health Organization; 1998.

21. Craig $C L$, Marshall AL, Sjostrom M, Bauman AE, Booth ML, Ainsworth BE et al. International Physical Activity Questionnaire: 12-Country Reliability and Validity. Med Sci Sports Exerc 2003;35:1381-95.

22. Hallal PC, Victora CG, Wells JC, Lima RC. Physical inactivity: prevalence and associated variables in Brazilian adults. Med Sci Sports Exerc 2003;35:1894-900.

23. Mion Júnior D, Pierin AMG, Bambirra AP, Assunção $\mathrm{JH}$, Monteiro JM, Chinen RY et al. Hypertension in employees of a university general hospital. Rev Hosp Clín 2004;59:329-336.

24. Cercato C, Mancini MC, Arguello AMC. Systemic hypertension, diabetes mellitus, and dyslipidemia in relation to body mass index: evaluation of a brazilian population. Rev Hosp Clín 2004;59:113-118.

\section{AGRADECIMENTOS}

A equipe da Unidade de Saúde da Família Dr. Milton Rabello I, em especial aos Agentes Comunitários de Saúde, pelo apoio na fase de coleta dos dados. A Prof $^{a}$ Juscélia Oliveira pela contribuição na revisão ortográfica e gramatical deste manuscrito.

\section{Endereço para correspondência}

Raildo da Silva Coqueiro

Rua Porto Alegre, 534, Patagônia.

CEP 45065-050. Vitória da Conquista, Bahia, Brasil

Recebido em 14/05/07

E-mail: raiconquista@yahoo.com.br 